\title{
Further evidence for the role of mode-independent short-term associations in spatial Simon effects
}

\author{
JAN DE HOUWER \\ Ghent University, Ghent, Belgium \\ TOM BECKERS \\ University of Leuven, Leuven, Belgium \\ and \\ STEFAAN VANDORPE and ROEL CUSTERS \\ Ghent University, Ghent, Belgium
}

\begin{abstract}
We examined the conditions under which short-term associations between stimuli and responses can produce spatial Simon effects. On location-relevant trials, participants gave neutral responses (i.e., they uttered the nonsense syllable "bee" or "boo") on the basis of whether the presented word had the meaning of "left" or "right." On location-irrelevant trials, they gave the same responses on the basis of the color of left and right squares. Performance on the location-irrelevant trials was affected by the match between the irrelevant location information and the location to which the correct response was assigned on the location-relevant trials. Experiment 1 showed that this extrinsic Simon effect was found only when the words on the location-relevant trials came from two different languages. In Experiment 2, we found an extrinsic Simon effect even when participants only received instructions about how to respond on location-relevant trials but no such trials were actually presented. Our findings suggest that task demands determine whether short-term associations are mode specific or mode independent and confirm that such associations can be set up as the result of instructions only.
\end{abstract}

In a typical spatial Simon study, participants are asked to press a left or right key on the basis of the color of a stimulus that is presented on the left or right side of a screen. Results consistently show that performance in such a task is better when the irrelevant location of the stimulus corresponds to the location of the correct response (e.g., press left in response to a stimulus on the left) than when the irrelevant location of the stimulus and that of the correct response differ (e.g., press left in response to a stimulus on the right). Similar results can be obtained when the irrelevant-location information is conveyed through modes other than physical location - for instance, when participants give spatial responses on the basis of the colors of arrows pointing to the left or right (symbolic mode) or the colors of words such as LEFT and RIGHT (verbal mode; see Simon, 1990, and Lu \& Proctor, 1995, for reviews). ${ }^{1}$ Spatial Simon effects are often attributed to the existence of long-term associations between nodes that represent the location conveyed by the stimulus on the one hand and the location of responses on the other (see, e.g., De

T.B. is a postdoctoral fellow of the FWO-Flanders (Belgium), and S.V. is a research assistant of the BOF (Ghent University, Belgium). Correspondence concerning this article should be addressed to J. De Houwer, Department of Psychology, Ghent University, Henri Dunantlaan 2, B-9000 Ghent, Belgium (e-mail: jan.dehouwer@ugent.be).
Jong, Liang, \& Lauber, 1994; Kornblum, Hasbroucq, \& Osman, 1990; Zorzi \& Umiltà, 1995; see Hommel, 1997, for a related proposal). Because activation can spread automatically along these associations, presenting a left (right) stimulus will result in the activation of a left (right) response, even when the location of the stimulus is irrelevant to the task.

Recent evidence, however, strongly suggests that shortterm associations also have an important impact on Simon effects. Whereas long-term associations are determined genetically or have resulted from overlearning during a lifetime, short-term associations are set up quickly as the result of instructions regarding the current task (see, e.g., Barber \& O'Leary, 1997; McClelland, McNaughton, \& O'Reilly, 1995; Tagliabue, Zorzi, Umiltà, \& Bassignani, 2000). The impact of these short-term associations on Simon effects is demonstrated by studies showing that Simon effects observed on location-irrelevant trials can be modulated by, and even based entirely upon, associations that were created as the result of task instructions. First, Simon effects can be eliminated or even reversed by the addition of location-relevant trials on which participants are asked to press a left key for right stimuli and a right key for left stimuli (incompatible mapping). This modulation occurs both when the location-relevant trials are presented before the (location-irrelevant) Simon trials (see, e.g., Tagliabue, Zorzi, \& Umiltà, 2002; Taglia- 
bue et al., 2000) and when the location-relevant and the Simon trials are intermixed (see, e.g., Proctor \& Lu, 1999; Proctor, Marble, \& Vu, 2000; see Proctor \& Vu, 2002, for a review). Second, De Houwer (2004) asked participants to utter the nonsense syllable "cale" or "cole" on the basis of the direction of arrows (i.e., left or right), the meaning of words (i.e., LEFT or RIGHT), and the color of squares presented left or right of the screen center. Thus, the words and arrows were the location-relevant stimuli and the squares were the location-irrelevant stimuli. Responses to squares were faster when the correct response was associated with the same location as the irrelevant location of the square than when the response was associated with a different location than the location of the square. For instance, when participants were asked to say "cale" in response to the word LEFT, a left arrow, and a green square, responses to the green square were faster when it was located on the left side of the screen than when it was located on the right side. This demonstrates that Simon effects can be found even with nonspatial responses that have been associated with left or right stimuli as the result of task instructions. Because the stimuli and responses are related extrinsically (i.e., because of task instructions during the experiment) rather than intrinsically (i.e., because of experiences before the onset of the experiment), we will refer to this kind of effects as extrinsic Simon effects (see also De Houwer, 2003a, 2003c).

At a theoretical level, these results imply that shortterm associations can be set up between stimulus and response representations and that activation can spread automatically along these associations. It is, however, not yet clear whether short-term associations are specific to the mode (e.g., verbal, symbolic, or physical location) in which location information is conveyed on the locationrelevant trials. On the one hand, Proctor et al. (2000) found a modulation of Simon effects when the stimuli on the location-relevant and -irrelevant trials were of the same mode but not when the stimuli were of different modes. For instance, in one of the conditions, participants were asked to press the left key in response to a white arrow pointing to the right and the right key in response to a white arrow pointing to the left (symbolic location-relevant trials). In a second condition, participants pressed the left key in response to the word RIGHT and the right key in response to the word LEFT (verbal location-relevant trials). In both conditions, they responded also on the basis of the colors of arrows that pointed to the left or the right (symbolic location-irrelevant trials). A reversed Simon effect (i.e., poorer performance when the irrelevant direction of the colored arrow corresponded to the position of the correct response) was found when arrows were presented on both the locationrelevant and the location-irrelevant trials. However, a standard Simon effect (i.e., better performance when the position of the colored arrow and that of the response matched) of normal magnitude was found when words were presented on the location-relevant trials and arrows on the location-irrelevant trials. Proctor et al. thus concluded that short-term associations are specific to the mode of the stimuli that are presented on the locationrelevant trials. On the other hand, in the study of De Houwer (2004), verbal stimuli (words) and symbolic stimuli (arrows) were presented on location-relevant trials, whereas location was conveyed by physical position on location-irrelevant trials (squares presented on the left or right). The fact that a Simon effect was found on the location-irrelevant trials suggests that an association was formed between the responses and mode-independent (e.g., semantic) representations of stimulus location.

De Houwer (2004) argued that the difference between his results and those of Proctor et al. (2000) might be related to the nature of the stimuli on the location-relevant trials. Whereas Proctor et al. presented only two stimuli on the location-relevant trials (e.g., only the words LEFT and RIGHT), De Houwer (2004) used four stimuli (i.e., the words LEFT and RIGHT, and left and right arrows). When there are four rather than two location-relevant stimuli, it could be more efficient to create two mode-independent short-term associations that involve the abstract representations of "left" and "right" rather than four separate short-term associations involving the perceptual representation of each stimulus.

However, it is also possible that the findings of De Houwer (2004) were due to the operation of modespecific rather than mode-independent short-term associations. In his study, location information was conveyed by physical position on the location-irrelevant trials and by both verbal and symbolic information on the locationrelevant trials. Proctor et al. (2000) pointed out that symbolic (i.e., direction of arrows) and physical (i.e., a square presented on the left or right) instantiations of location are both visuospatial in nature. As a result, information about physical location (e.g., presentation of a left or right square) could result in a partial activation of mode-specific short-term associations that involve symbolic representations. Therefore, the effect that De Houwer (2004) observed might not have been based on modeindependent short-term associations after all. If this argument is correct, one would predict that the effect would disappear when only words are used on the location-relevant trials.

We therefore further examined the role and nature of short-term associations in Simon tasks. Experiment 1 was a replication of De Houwer (2004) except for the fact that only verbal stimuli were presented on the locationrelevant trials. This allowed us to exclude the alternative explanation of the results of De Houwer (2004) in terms of partial activation of mode-specific short-term associations. We also tested the hypothesis that mode-independent short-term associations are more likely to operate when each location is instantiated by more than one stimulus. In the Dutch-only condition, only the Dutch words for "left" and "right" were presented; mode-specific associations can be formed between the perceptual representation of these words on the one hand and the representa- 
tions of the responses on the other. In the Dutch-French condition, both the Dutch (LINKS, RECHTS) and the French (GAUCHE, DROITE) words for "left" and "right" were presented. In this case, it would be more efficient for our bilingual Dutch-French-speaking participants to create mode-independent associations between the abstract, semantic representations of "left" and "right" on the one hand and the representations of response on the other. We thus predicted that an extrinsic Simon effect would be found only in the condition in which Dutch and French words were presented on the location-relevant trials.

In a second experiment, we tried to obtain stronger evidence for the claim that it is indeed short-term rather than long-term associations that form the basis of extrinsic Simon effects. As we pointed out earlier, it is generally assumed that long-term associations develop slowly as a result of practice, whereas short-term associations are set up quickly as a result of task instructions (see, e.g., Barber \& O'Leary, 1997; McClelland et al., 1995; Tagliabue et al., 2000). Importantly, all studies that provided evidence for the role of short-term associations included location-relevant trials on which participants could practice the stimulus-response associations. One could argue that new long-term associations were formed as the result of practice on location-relevant trials and that these long-term associations were responsible for the observed effects. De Houwer (2004) pointed out that such an account is unlikely, because he found an extrinsic Simon effect also during a practice block that consisted exclusively of location-irrelevant trials and that was presented after an initial practice block of only 20 location-relevant trials. However, one could still argue that 20 location-relevant practice trials are sufficient to establish long-term stimulus-response associations. To rule out such potential arguments, we replicated the experiment of De Houwer (2004) but now only instructed participants on how to respond on location-relevant trials without actually presenting such trials. If an extrinsic Simon effect is found under these conditions, it would provide even stronger evidence for the fact that shortterm associations can produce spatial Simon effects.

\section{EXPERIMENT 1}

\section{Method}

Participants. Twenty-nine first-year psychology students at Ghent University took part in this experiment in exchange for course credit. They were native Dutch speakers who were born in Belgium (a trilingual Dutch-French-German-speaking country) and had been taught French from primary school onward, starting on average at the age of 10 years. Fifteen participants were randomly assigned to the Dutch-only condition. The other 14 participants were assigned to the Dutch-French condition.

Stimuli and Apparatus. The blue and green squares that were presented on the location-irrelevant trials were $9 \times 9 \mathrm{~mm}$. They appeared $1.5 \mathrm{~cm}$ to the left or to the right of the screen center. On the location-relevant trials, the Dutch and French words for "left" (LINKS, GAUCHE) and "right" (RECHTS, DROITE) were presented at screen center in white uppercase letters $7 \mathrm{~mm}$ high and $6 \mathrm{~mm}$ wide. All stimuli were presented on a black background. A voice key that was connected to the game port of a computer registered the verbal responses. Presentation of the stimuli and registration of the responses were controlled by an adaptation of the Turbo Pascal 5.0 program that had been used by De Houwer (2004). The program was implemented on an IBM-compatible 486 PC with a 14-in. screen.

Procedure. The participants were tested individually in a dimly lit room and were seated approximately $50 \mathrm{~cm}$ from the computer screen. Instructions that appeared on the screen informed the participants that words and colored squares would be presented one by one. Their task was to utter the nonsense syllable "bee" or "boo" depending on the meaning of the word or the color of the square. The 15 participants who were assigned to the Dutch-only condition were told that the words would be the Dutch words LINKS (left) and RECHTS (right). The other participants were assigned to the Dutch-French condition and were told that the Dutch and French words LINKS and GAUCHE (left), and RECHTS and DROITE (right), would be presented one by one on the screen. Approximately half of the participants in each condition were instructed to say "bee" to words meaning "left" and "boo" to words meaning "right." The reverse was true for the other participants. Orthogonally to these manipulations, approximately half of the participants were instructed to say "bee" in response to blue squares and "boo" in response to green squares, whereas the color-response assignments were reversed for the other participants. Finally, the participants were informed that incorrect responses would be indicated by a short beep and were told that the experiment would consist of three series of practice trials and four series of test trials. We chose to present error feedback in order to prevent the participants from forgetting or switching the arbitrary stimulus-response assignments.

All the participants first completed a practice block of 20 trials on which only words were presented. In the Dutch-only condition, each Dutch word appeared on 10 trials. In the Dutch-French condition, each of the four words was presented on five trials. During the second practice block of 20 trials, the blue and green squares were each presented 5 times on the left side of the screen and 5 times on the right side. A third practice block also consisted of 20 trials. These trials were the first of a series of 24 trials during which each Dutch word was presented 6 times (Dutch-only condition) or each Dutch word and each French word was presented 3 times (Dutch-French condition), and each colored square was presented 3 times on the left and 3 times on the right. The final four trials of this series were used as warm-up trials (see below). Next, two blocks of 60 experimental trials were presented. In each block, the two Dutch words each appeared 10 times (Dutch-only condition), or the four words each appeared 5 times (Dutch-French condition), whereas the green and blue squares were each presented 10 times on the left side and 10 times on the right side. The trials within each practice block and each experimental block were presented in a semirandom order that was determined separately for each block and each participant. The following restrictions applied: (1) The stimulus could not be the same on more than two consecutive trials and (2) the correct response could not be the same on four or more consecutive trials. The participants could take a break for as long as they wished after reading the instructions, after each practice block, and after each series of 30 experimental trials. During this break, which rarely lasted longer than $30 \mathrm{sec}$, instructions appeared on the screen that informed the participants about whether the next series of trials would be practice or test and about the nature of the stimuli that would appear during that series (i.e., words only, squares only, or all stimuli). A warm-up trial immediately preceded each of the four series of 30 experimental trials.

Each of the practice, warm-up, and experimental trials started with the presentation of a fixation cross for $500 \mathrm{msec}$, immediately followed by a stimulus that stayed on the screen until the voice key registered a response or 3,000 msec had elapsed. The experimenter then coded the participant's response or entered a separate code if the voice key did not accurately register the verbal response. If the 
response was incorrect, a $200-\mathrm{Hz}$ tone was presented for $250 \mathrm{msec}$. The next trial started $1,500 \mathrm{msec}$ after the experimenter entered the code or after the end of the feedback tone.

\section{Results}

Experimental trials. We first analyzed reaction times (RTs) and percentage of errors on the location-irrelevant experimental trials. Trials on which RTs were shorter than $200 \mathrm{msec}$ or longer than $2,000 \mathrm{msec}(0.26 \%$ of all trials) and trials on which the voice key did not accurately register the response ( $1.85 \%$ of all trials) were excluded from the analyses. We also ignored RTs on trials in which an incorrect response was given $(3.78 \%$ of the remaining trials). The data of 1 participant were excluded because she gave an incorrect response on $18 \%$ of all location-irrelevant trials, which was more than three standard deviations higher than the mean percentage of errors of the total group. Means were submitted to analyses of variance (ANOVAs) with stimulus location (square on the left or on the right) and associated response location (correct response was also assigned to words with the meaning "left" or to words with the meaning "right") as within-subjects variables and condition (Dutch-only or Dutch-French) as a between-subjects variable. The data of the two conditions were then analyzed separately using stimulus location $\times$ associated response location ANOVAs.

The overall ANOVA of the RT data revealed a significant three-way interaction $[F(1,26)=4.64, p=.04]$. The follow-up ANOVAs showed that the crucial interaction between stimulus location and associated response location was significant in the Dutch-French condition $[F(1,13)=7.11, p=.02]$ but not in the Dutchonly condition $(F<1)$. Table 1 shows that RTs were shorter when the correct response was associated with the location at which the square was presented, but only in the Dutch-French condition. The ANOVAs did not reveal any other effects (all $F \mathbf{s}<1)$ except for a marginally significant interaction between stimulus location and associated response location in the overall ANOVA $[F(1,26)=2.97, p=.10]$.

Table 1

Mean Reaction Time (RT, in Milliseconds) and Percentage of Errors on Location-Irrelevant Trials of the Experimental Phase in Experiment 1 as a Function of Stimulus Location, Associated Response Location, and Condition

\begin{tabular}{|c|c|c|c|c|c|c|c|c|}
\hline \multirow{4}{*}{$\begin{array}{l}\text { Stimulus } \\
\text { Location }\end{array}$} & \multicolumn{8}{|c|}{ Associated Response Location } \\
\hline & \multicolumn{4}{|c|}{ Left } & \multicolumn{4}{|c|}{ Right } \\
\hline & \multicolumn{2}{|c|}{ RT } & \multicolumn{2}{|c|}{$\%$ Error } & \multicolumn{2}{|c|}{ RT } & \multicolumn{2}{|c|}{ \% Error } \\
\hline & $M$ & $S D$ & $M$ & $S D$ & $M$ & $S D$ & $M$ & $S D$ \\
\hline \multicolumn{9}{|c|}{ Dutch-Only Condition } \\
\hline Left & 631 & 115 & 2.16 & 2.59 & 626 & 113 & 2.54 & 3.35 \\
\hline Right & 621 & 115 & 2.50 & 3.80 & 621 & 115 & 2.91 & 3.87 \\
\hline \multicolumn{9}{|c|}{ Dutch-French Condition } \\
\hline Left & 628 & 131 & 2.64 & 5.00 & 646 & 145 & 2.52 & 3.81 \\
\hline Right & 652 & 143 & 7.27 & 6.97 & 624 & 147 & 3.65 & 4.64 \\
\hline
\end{tabular}

The same analyses were conducted on the error data. In the overall analysis, neither the interaction between stimulus location and associated response location $[F(1,26)=2.07, p=.16]$ nor the three-way interaction $[F(1,26)=2.16, p=.15]$ was significant. Separate analyses showed, however, that the crucial stimulus location $\times$ associated response location interaction was significant in the Dutch-French condition $[F(1,13)=$ $5.97, p=.03$ ] but not in the Dutch-only condition $(F<1)$. Table 1 shows that the pattern of results paralleled that of the RT data. The ANOVAs did not reveal any other effects (all $F_{\mathrm{S}}<2.38$ ) except for a main effect of stimulus location in the overall ANOVA $[F(1,26)=$ 5.93, $p=.02]$ and in the analysis of the Dutch-French condition $[F(1,13)=7.23, p=.02]$, and a marginally significant interaction of stimulus location and condition in the overall ANOVA $[F(1,26)=3.61, p=.07]$.

Practice trials. In accordance with De Houwer (2004), we also analyzed the data from the second practice phase, which consisted of only 20 location-irrelevant trials. We again excluded trials on which the RT was shorter than $200 \mathrm{msec}$ or longer than $2,000 \mathrm{msec}(0.70 \%$ of all trials), the voice key failed to accurately register the response $(2.60 \%$ of all trials), or an incorrect response was given (6.93\% of the remaining trials). These data were analyzed in the same way as the data of the location-irrelevant experimental trials.

Confirming the findings of De Houwer (2004), the overall ANOVA on the RT data revealed a significant interaction between stimulus location and associated response location $[F(1,27)=5.52, p=.03]$. RTs on locationirrelevant trials were shorter when the irrelevant location of the stimulus was the same as the location that was associated with the response (see Table 2). This effect, however, was not modulated by condition $(F<1)$. Separate analyses showed that the interaction between stimulus location and associated response location was significant when only Dutch words were presented $[F(1,14)=8.22$, $p=.01]$ but not when both Dutch and French words were presented $[F(1,13)=1.35, p=.27]$. The analyses did not reveal any other effects (all $F \mathrm{~s}<2.38$ ) except for a marginally significant interaction between stimulus location and condition in the overall analysis $[F(1,27)=3.72$, $p=.07]$.

The overall analysis of the percentage of errors on the location-irrelevant practice trials did not reveal an interaction between stimulus location and associated response location $(F<1)$ but did show a marginally significant three-way interaction $[F(1,27)=2.89, p=.10]$. Separate analyses showed that the interaction between stimulus location and associated response location was significant neither in the Dutch-only condition $[F(1,14)=$ $2.50, p=.14]$ nor in the Dutch-French condition $(F<1)$. An inspection of Table 2 shows, however, that the effect tended to be in the expected direction in the Dutch-French condition (i.e., fewer errors when stimulus location and associated response location matched) and in the opposite direction in the Dutch-only condition (i.e., fewer er- 
Table 2

Mean Reaction Time (RT, in Milliseconds) and Percentage of Errors on Location-Irrelevant Trials of the Practice Phase in Experiment 1 as a Function of Stimulus Location, Associated Response Location, and Condition

\begin{tabular}{|c|c|c|c|c|c|c|c|c|}
\hline \multirow{4}{*}{$\begin{array}{l}\text { Stimulus } \\
\text { Location }\end{array}$} & \multicolumn{8}{|c|}{ Associated Response Location } \\
\hline & \multicolumn{4}{|c|}{ Left } & \multicolumn{4}{|c|}{ Right } \\
\hline & \multicolumn{2}{|c|}{ RT } & \multicolumn{2}{|c|}{$\%$ Error } & \multicolumn{2}{|c|}{ RT } & \multicolumn{2}{|c|}{$\%$ Error } \\
\hline & $M$ & $S D$ & $M$ & $S D$ & $M$ & $S D$ & $M$ & $S D$ \\
\hline \multicolumn{9}{|c|}{ Dutch-Only Condition } \\
\hline Left & 583 & 128 & 8.57 & 12.92 & 666 & 182 & 5.71 & 9.38 \\
\hline Right & 594 & 131 & 0.00 & 0.00 & 578 & 82 & 4.29 & 8.52 \\
\hline \multicolumn{9}{|c|}{ Dutch-French Condition } \\
\hline Left & 575 & 111 & 10.00 & 13.01 & 677 & 190 & 12.86 & 18.58 \\
\hline Right & 669 & 197 & 7.14 & 9.95 & 676 & 210 & 4.29 & 8.52 \\
\hline
\end{tabular}

rors when stimulus location and associated response location differed). This suggests that the significant Simon effect in the RT data of the Dutch-only condition could have been due partially to a speed-accuracy tradeoff. The analyses of the error data did not reveal any other effects (all $F_{\mathrm{S}}<1$ ) except for a main effect of stimulus location that emerged in the overall analyses $[F(1,27)=$ $11.65, p=.002]$, the analysis of the Dutch-only condition $[F(1,14)=7.88, p=.01]$, and the analysis of the Dutch-French condition $[F(1,13)=4.43, p=.05]$. In all three cases, more errors were made in response to squares on the left than in response to squares on the right (see Table 2). Note, however, that the overall analysis and the analysis in the Dutch-only condition were compromised because no errors were made in the Dutchonly condition when the square was on the right and the response was associated with left.

\section{Discussion}

Like De Houwer (2004), we found a clear extrinsic Simon effect in the RT data of the experimental trials. This replication is important not only because it attests to the reliability of the results of De Houwer (2004), but also because it provides stronger evidence for the existence of mode-independent short-term associations than the original data did. Because physical and symbolic representations of location are both visuospatial in nature, it is possible that squares on the left or right of the screen partially activate mode-specific short-term associations that are set up for responding to left and right arrows. Because De Houwer (2004) presented arrows on location-relevant trials, such a partial activation of mode-specific short-term associations could have produced the Simon effect that he observed. In the present experiment, however, only words were presented on location-relevant trials. Therefore, only mode-independent short-term associations could have produced the observed Simon effect.

Moreover, the data strongly suggest that such modeindependent associations are set up only when each location is represented by more than one stimulus on the location-relevant trials. In the Dutch-only condition, only the Dutch words for "left" and "right" were presented on the location-relevant trials, whereas in the Dutch-French condition each location was represented by both the Dutch word and the French word for that location. Results showed that the extrinsic Simon effect on the experimental trials was larger and significant only in the Dutch-French condition. This finding clarifies why De Houwer (2004) found evidence for mode-independent short-term associations. As was the case in our experiment, De Houwer (2004) used two location-relevant stimuli for each location. Furthermore, the present results also suggest that people are very flexible in setting up short-term associations. When each location is instantiated by two location-relevant stimuli, it is more efficient to respond on the basis of the semantic characteristics of the location-relevant stimuli. As a result, mode-independent associations can be set up that involve the abstract representations of "left" and "right." These associations can therefore also be activated by the spatial properties of other stimuli even though these spatial properties are irrelevant and are conveyed in a different mode. When each location is instantiated by only one location-relevant stimulus, there is no need for semantic processing of the location-relevant stimuli. Rather, it suffices to have associations that involve lowlevel perceptual representations (e.g., representations at the level of letters when the correct response can be selected on the basis of the first letter of the word). Such associations are therefore mode-specific and cannot be activated by stimuli that convey spatial information in another mode.

During the practice trials, a significant extrinsic Simon effect was found in RT data of the Dutch-only condition. This suggests that mode-independent shortterm associations can operate even when there is only one location-relevant stimulus for each location. One should note, however, that the observed effect seemed to be at least partially due to a speed-accuracy tradeoff. Moreover, it was clearly short-lived, given that no extrinsic Simon effect was found during the subsequent experimental trials in the Dutch-only condition. Assuming that the effect was genuine, one could argue that it was due to the fact that, during the instructions, all the participants were asked to pay attention to the (abstract) meaning of the words. At some later point in time, however, the participants might have realized that they could also respond on the basis of the perceptual features of the two locationrelevant words. As a result, mode-specific short-term associations that involved low-level perceptual representations could have become active. Although speculative, this account is also in line with the idea that people are very flexible in setting up short-term associations.

\section{EXPERIMENT 2}

The aim of our second experiment was to provide an even more rigorous test of the hypothesis that Simon effects can be based on short-term rather than long-term 
associations. In all previous studies on the influence of short-term associations on RT performance, participants were not only instructed on how to respond to locationrelevant stimuli but also actually responded to such stimuli. One could argue that such a practice is sufficient to create long-term associations that could produce or modulate Simon effects. In an attempt to counter such arguments, we ran an experiment in which participants only received instruction about how to respond to locationrelevant stimuli.

\section{Method}

Participants. Forty first-year psychology students at Ghent University took part in exchange for course credit. One of them had participated in Experiment 1.

Stimuli, Apparatus, and Procedure. This experiment was identical to Experiment 1 except on the following points. First, only location-irrelevant stimuli were presented. Second, at the start of the experiment, the participants read written instructions that informed them that the Dutch words LINKS (left) and RECHTS (right), a left and a right arrow, and blue and green squares would appear on the screen. They were then given instructions on how to respond to the locationrelevant (words and arrows) and location-irrelevant (squares) stimuli. Afterward, the participants were told that the responses to the words and arrows would be especially important. Because only very few words and arrows would be presented, it was important not to forget how to respond to these stimuli. Rather, they should always keep in mind what they should do if a word or arrow appeared so that they could react rapidly to those stimuli. Finally, the participants were asked to take some time to memorize the stimulus-response rules. They were told that previous research had indicated that this would help them perform the task.

A third difference with Experiment 1 was that 8 location-irrelevant warm-up trials were presented immediately after the instructions. On these practice trials, each colored square was presented two times on the left and two times on the right in the same way as in Experiment 1 . These 8 practice trials were followed immediately by 40 experimental location-irrelevant trials on which each colored square was presented 10 times on the left side and 10 times on the right side of the screen (see Experiment 1).

\section{Results and Discussion}

We calculated the mean RT and percentage of errors on the 40 experimental location-irrelevant trials. As in Experiment 1, trials on which the RT was shorter than $200 \mathrm{msec}$ or longer than $2,000 \mathrm{msec}$ were excluded from the analyses ( $0.38 \%$ of all trials), as were trials on which the voice key did not accurately register the response (3.38\% of all trials). We also ignored RTs on trials on which an incorrect response was given $(2.99 \%$ of the remaining trials). Means were analyzed using ANOVAs with stimulus location (square on the left or on the right) and associated response location (correct responses were also assigned to the word LINKS and a left arrow and to the word RECHTS and a right arrow) as within-subjects variables. All relevant means can be found in Table 3.

The ANOVA of the RT data revealed a significant interaction between stimulus location and associated response location $[F(1,39)=6.66, p=.01]$. Table 3 shows that responses were faster when the location of the stimulus and the associated location of the response matched
Table 3

Mean Reaction Time (RT, in Milliseconds) and Percentage of Errors on Location-Irrelevant Trials of the Experimental Phase in Experiment 2 as a Function of Stimulus Location and Associated Response Location

\begin{tabular}{|c|c|c|c|c|c|c|c|c|}
\hline \multirow{5}{*}{$\begin{array}{l}\text { Stimulus } \\
\text { Location }\end{array}$} & \multicolumn{8}{|c|}{ Associated Response Location } \\
\hline & \multicolumn{8}{|c|}{ Associated Response Location } \\
\hline & \multicolumn{4}{|c|}{ Left } & \multicolumn{4}{|c|}{ Right } \\
\hline & \multicolumn{2}{|c|}{ RT } & \multicolumn{2}{|c|}{$\%$ Error } & \multicolumn{2}{|c|}{ RT } & \multicolumn{2}{|c|}{$\%$ Error } \\
\hline & $M$ & $S D$ & $M$ & $S D$ & $M$ & $S D$ & $M$ & $S D$ \\
\hline Left & 535 & 115 & 3.83 & 6.85 & 574 & 134 & 2.79 & 5.10 \\
\hline Right & 560 & 118 & 2.81 & 5.14 & 558 & 120 & 2.44 & 4.70 \\
\hline
\end{tabular}

than when they differed. The main effect of stimulus location was not significant $(F<1)$, but the main effect of response was $[F(1,39)=4.22, p=.05]$, showing that responses that were associated with "left" were emitted more quickly than responses associated with "right." The ANOVA of the error data did not reveal any effects (all $\left.F_{\mathrm{S}}<1\right)$.

We again found a significant extrinsic Simon effect, even though no location-relevant trials were presented. This provides strong evidence for the claim that Simon effects can be based on short-term associations - that is, associations that are set up as the result of task instructions.

\section{GENERAL DISCUSSION}

Even though the spatial Simon effect has been studied for many years, the impact of short-term associations on this effect was recognized only recently (see, e.g., Proctor et al., 2000; Tagliabue et al., 2000). We used the extrinsic Simon task to examine whether such short-term associations are mode specific or mode independent and tried to obtain stronger evidence for the claim that it is indeed short-term associations that underlie extrinsic Simon effects.

In Experiment 1, the participants said "bee" or "boo" on the basis of whether a word referred to "left" or to "right" (location-relevant trials) and whether a left or a right square was colored green or blue (location-irrelevant trials). Responses were faster when the irrelevant location of the square matched the location that was associated with the correct response (e.g., saying "bee" to a square on the left when "bee" was also the correct response for words with the meaning "left"). Such an extrinsic Simon effect emerged when the Dutch and French words for "left" and "right" were presented on the locationrelevant trials, but it was not found when only the Dutch words were used. This result suggests that De Houwer (2004) found evidence for mode-independent short-term associations because he used two stimuli (i.e., a word and an arrow) for each location on the location-relevant trials. Moreover, the design used by De Houwer (2004) did not exclude the possibility that the effects were due to mode-specific associations, whereas we used an improved design that eliminates this possibility. 
Our data thus provide strong evidence for the claim that short-term associations can be mode independent and clarify the conditions under which mode-independent associations are established. We did, however, look only at situations in which there were no long-term associations between the responses and spatial location. In experiments such as those of Proctor et al. (2000), locationrelevant stimuli were mapped on incompatible responses (e.g., press left for right stimuli or press right for left stimuli). In our study, on the other hand, responses (i.e., "bee" or "boo") were unrelated to spatial position. One could argue that the presence of conflicting long-term associations encourages the formation of short-term associations that involve mode-specific levels of representation. If this is the case, one would never expect a mode-independent modulation of Simon effects by an incompatible location-relevant task, even when there is more than one location-relevant stimulus for each location. It thus remains to be seen whether evidence for mode-independent associations can be found when there are conflicting long-term associations.

The results of Experiment 2 provide strong evidence for the claim that Simon effects can be based on short-term associations only. In Experiment 2, the participants were only instructed on how to respond to location-relevant trials but were not actually given trials on which the location of the stimulus was relevant. Because the participants could therefore never practice the new location-response mappings, it is reasonable to argue that new long-term associations could not have formed. This strongly suggests that the observed extrinsic Simon effect was due to the operation of short-term associations that were formed on the basis of instructions only.

Our data have clear theoretical implications. They demonstrate more clearly than ever before that models of the Simon effect (and other stimulus-response compatibility effects; see De Houwer, 2003b) should allow for an automatic influence of short-term associations on performance. Until now, the few models that do allow for such an influence assume either that these associations are mode specific (e.g., Cohen, Dunbar, \& McClelland, 1990) or that they are mode independent (e.g., Zhang \& Kornblum, 1998). Our results suggest that the cognitive system is much more flexible than that. Short-term associations can involve either mode-specific or modeindependent representations. Whether associations are mode specific or mode independent seems to depend on the task demands. When it is more efficient for participants to focus on the abstract meaning of stimuli (as is the case when each relevant location is instantiated by several stimuli), short-term associations are mode independent. When it is more efficient to focus on lowlevel perceptual features (as is the case when there is just one stimulus for each relevant location), short-term associations appear to be mode specific. Therefore, models need to be adjusted in such a way that they acknowledge the flexible nature of short-term associations.

\section{REFERENCES}

BARBER, P., \& O'LEARY, M. (1997). The relevance of salience: Towards an activational account of irrelevant stimulus-response compatibility effects. In B. Hommel \& W. Prinz (Eds.), Theoretical issues in stimulus-response compatibility (pp. 135-172). Amsterdam: Elsevier, North-Holland.

Cohen, J. D., Dunbar, K., \& McClelland, J. L. (1990). On the control of automatic processes: A parallel distributed processing account of the Stroop effect. Psychological Review, 97, 332-361.

De Houwer, J. (2003a). The extrinsic affective Simon task. Experimental Psychology, 50, 77-85.

De Houwer, J. (2003b). On the role of stimulus-response and stimulusstimulus compatibility in the Stroop effect. Memory \& Cognition, 31, 353-359.

DE Houwer, J. (2003c). A structural analysis of indirect measures of attitudes. In J. Musch \& K. C. Klauer (Eds.), The psychology of evaluation: Affective processes in cognition and emotion (pp. 219-244). Mahwah, NJ: Erlbaum.

De Houwer, J. (2004). Spatial Simon effects with nonspatial responses. Psychonomic Bulletin \& Review, 11, 49-53.

De Jong, R., Liang, C. C., \& Lauber, E. (1994). Conditional and unconditional automaticity: A dual-process model of effects of spatial S-R correspondence. Journal of Experimental Psychology: Human Perception \& Performance, 20, 731-750.

Hommel, B. (1997). Toward an action-concept model of stimulusresponse compatibility. In B. Hommel \& W. Prinz (Eds.), Theoretical issues in stimulus-response compatibility (pp. 281-320). Amsterdam: Elsevier, North-Holland.

Kornblum, S., HasbroucQ, T., \& Osman, A. (1990). Dimensional overlap. Cognitive basis for stimulus-response compatibility: A model and taxonomy. Psychological Review, 97, 253-270.

Lu, C.-H., \& Proctor, R. W. (1995). The influence of irrelevant location information on performance: A review of the Simon and spatial Stroop effects. Psychonomic Bulletin \& Review, 2, 174-207.

McClelland, J. L., McNaughton, B. L., \& O'Reilly, R. C. (1995). Why there are complementary learning systems in the hippocampus and neocortex: Insights from the successes and failures of connectionist models of learning and memory. Psychological Review, 102, 419-437.

Proctor, R. W., \& Lu, C.-H. (1999). Processing irrelevant location information: Practice and transfer effects in choice-reaction tasks. Memory \& Cognition, 27, 63-77.

Proctor, R. W., Marble, J. G., \& Vu, K.-P. L. (2000). Mixing incompatibly mapped location-relevant trials with location-irrelevant trials: Effects of stimulus mode in the reverse Simon effect. Psychological Research, 64, 11-24.

Proctor, R. W., \& Vu, K.-P. L. (2002). Eliminating, magnifying, and reversing spatial compatibility effects with mixed location-relevant and -irrelevant trials. In W. Prinz \& B. Hommel (Eds.), Common mechanisms in perception and action: Attention and performance $X I X$ (pp. 443-473). Oxford: Oxford University Press.

Simon, J. R. (1990). The effects of an irrelevant directional cue on human information processing. In R. W. Proctor \& T. G. Reeve (Eds.), Stimulus-response compatibility: An integrated perspective (pp. 31-86). Amsterdam: North-Holland.

Tagliabue, M., Zorzi, M., \& Umiltà, C. (2002). Cross-modal remapping influences the Simon effect. Memory \& Cognition, 30, 18-23.

Tagliabue, M., Zorzi, M., Umiltà, C., \& Bassignani, F. (2000). The role of long-term-memory and short-term-memory links in the Simon effect. Journal of Experimental Psychology: Human Perception \& Performance, 26, 648-670.

Zhang, H., \& Kornblum, S. (1998). The effects of stimulus-response mapping and irrelevant stimulus-response and stimulus-stimulus overlap in four-choice Stroop tasks with single-carrier stimuli. Journal of Experimental Psychology: Human Perception \& Performance, 24, 3-19.

ZorZi, M., \& Umiltà, C. (1995). A computational model of the Simon effect. Psychological Research, 58, 193-205. 


\section{NOTE}

1. We thus distinguish between mode and sensory modality. Mode refers to the form in which information is conveyed (e.g., verbal, physical, or symbolic), whereas modality refers to the sensory channel through which the information is received (e.g., visual or auditory). The fact that mode and modality need to be distinguished is evidenced by the fact that, within each modality, information can be conveyed in different modes and vice versa. For instance, presenting squares on the left or right side of a screen conveys visual information about spatial location in a physical mode, whereas visually presenting the words LEFT and RIGHT conveys visual information about spatial location in a verbal mode. Note that Tagliabue, Zorzi, and Umiltà (2002) found that the effect of short-term associations can be independent of sensory modality. They did not, however, examine the impact of mode.

(Manuscript received June 16, 2003; revision accepted for publication August 23, 2004.) 